\title{
TWISTED LUBIN-TATE FORMAL GROUP LAWS, RAMIFIED WITT VECTORS AND (RAMIFIED) ARTIN-HASSE EXPONENTIALS \\ BY
}

\author{
MICHIEL HAZEWINKEL
}

\begin{abstract}
For any ring $R$ let $\Lambda(R)$ denote the multiplicative group of power series of the form $1+a_{1} t+\cdots$ with coefficients in $R$. The Artin-Hasse exponential mappings are homomorphisms $W_{p, \infty}(k) \rightarrow \Lambda\left(W_{p, \infty}(k)\right)$, which satisfy certain additional properties. Somewhat reformulated, the Artin-Hasse exponentials turn out to be special cases of a functorial ring homomorphism $E: W_{p, \infty}(-) \rightarrow$ $W_{p, \infty}\left(W_{p, \infty}(-)\right)$, where $W_{p, \infty}$ is the functor of infinite-length Witt vectors associated to the prime $p$. In this paper we present ramified versions of both $W_{p, \infty}(-)$ and $E$, with $W_{p, \infty}(-)$ replaced by a functor $W_{q, \infty}^{F}(-)$, which is essentially the functor of $q$-typical curves in a (twisted) Lubin-Tate formal group law over $A$, where $A$ is a discrete valuation ring that admits a Frobenius-like endomorphism $\sigma$ (we require $\sigma(a) \equiv a^{q} \bmod m$ for all $a \in A$, where $\mathfrak{m}$ is the maximal idea of $A$ ). These ramified-Witt-vector functors $W_{q, \infty}^{F}(-)$ do indeed have the property that, if $k=$ $A / \mathrm{m}$ is perfect, $A$ is complete, and $l / k$ is a finite extension of $k$, then $W_{q, \infty}^{F}(l)$ is the ring of integers of the unique unramified extension $L / K$ covering $l / k$.
\end{abstract}

1. Introduction. For each ring $R$ (commutative with unit element 1 ) let $\Lambda(R)$ be the abelian group of power series of the form $1+r_{1} t+r_{2} t^{2}+\cdots$. Let $W_{p, \infty}(R)$ be the ring of Witt vectors of infinite length associated to the prime $p$ with coefficients in $R$. Then the "classical" Artin-Hasse exponential mapping is a map $E: W_{p, \infty}(k) \rightarrow \Lambda\left(W_{p, \infty}(k)\right)$ defined for all perfect fields $k$ as follows (cf. e.g. [1] and [13]). Let $\Phi(y)$ be the power series

$$
\Phi(y)=\prod_{(p, n)=1}\left(1-y^{n}\right)^{\mu(n) / n},
$$

where $\mu(n)$ is the Möbius function. Then $\Phi(y)$ has its coefficients in $\mathbf{Z}_{p}$, cf. e.g. [13]. Because $k$ is perfect every element of $W_{p, \infty}(k)$ can be written in the form $\mathbf{b}=\sum_{i=1}^{\infty} \tau\left(c_{i}\right) p^{i}$, with $c_{i} \in k$, and $\tau: k \rightarrow W_{p, \infty}(k)$ the unique system of multiplicative representatives. One now defines

$$
E: W_{p, \infty}(k) \rightarrow \Lambda\left(W_{p, \infty}(k)\right), \quad E(\mathbf{b})=\prod_{i=0}^{\infty} \Phi\left(\tau\left(c_{i}\right) t\right)^{p^{\prime}} .
$$

Now let $W(-)$ be the ring functor of big Witt vectors. Then $W(-)$ and $\Lambda(-)$ are isomorphic, the isomorphism being given by $\left(a_{1}, a_{2}, \ldots\right) \mapsto \prod_{i=1}^{\infty}\left(1-a_{i} t^{i}\right)$, cf. [2]. Now there is a canonical quotient map $W(-) \rightarrow W_{p, \infty}(-)$ and composing $E$ with $\Lambda(-) \simeq W(-)$ and $W(-) \rightarrow W_{p, \infty}(-)$ we find an Artin-Hasse exponential $E$ : $W_{p, \infty}(k) \rightarrow W_{p, \infty}\left(W_{p, \infty}(k)\right)$. 
1.1. THEOREM. There exists a unique functorial homomorphism of ring-valued functors $E: W_{p, \infty}(-) \rightarrow W_{p, \infty}\left(W_{p, \infty}(-)\right)$ such that for all $n=0,1,2, \ldots, w_{p, n} \circ E$ $=f^{n}$, where $f$ is the Frobenius endomorphism of $W_{p, \infty}(-)$ and where $w_{p, n}$ : $W_{p, \infty}\left(W_{p, \infty}(-)\right) \rightarrow W_{p, \infty}(-)$ is the ring homomorphism which assigns to the sequence $\left(\mathbf{b}_{0}, b_{1}, \ldots\right)$ of Witt vectors the Witt vector $b_{0}^{p^{n}}+p b_{1}^{p^{n-1}}+\cdots+p^{n-1} b_{n-1}^{p}+$ $p^{n} \mathbf{b}_{n}$.

It should be noted that the classical definition of $E$ given above works only for perfect fields of characteristic $p>0$. In this form Theorem 1.1 is probably due to Cartier, cf. [5].

Now let $A$ be a complete discrete valuation ring with residue field of characteristic $p$, such that there exist a power $q$ of $p$ and an automorphism $\sigma$ of $K$, the quotient field of $A$, such that $\sigma(a) \equiv a^{q} \bmod \mathfrak{m}$ for all $a \in A$, where $\mathfrak{m}$ is the maximal ideal of $A$. It is the purpose of the present paper to define ramified Witt vector functors $W_{q, \infty}^{F}(-): \mathbf{A l g}_{A} \rightarrow \mathbf{A l g}_{A}$, where $\mathbf{A l g}_{A}$ is the category of $A$-algebras, and a ramified Artin-Hasse exponential mapping $E: W_{q, \infty}^{F}(-) \rightarrow W_{q, \infty}^{F}\left(W_{q, \infty}^{F}(-)\right)$.

There is such a ramified-Witt-vector functor $W_{q, \infty}^{F}$ associated to every twisted Lubin-Tate formal group law $F(X, Y)$ over $A$. This last notion is defined as follows. Let $f(X)=X+a_{2} X^{2}+\cdots \in K[[X]]$ and suppose that $a_{i} \in A$ if $q$ does not divide $i$ and $a_{q i}-\omega^{-1} \sigma\left(a_{i}\right) \in A$ for all $i$ for a certain fixed uniformizing element $\omega$. Then $F(X, Y)=f^{-1}(f(X)+f(Y)) \in A[[X, Y]]$, and the formal group laws thus obtained are what we call twisted Lubin-Tate group laws. The Witt-vector functors $W_{q, \infty}^{F}(-)$ for varying $F$ are isomorphic if the formal group laws are strictly isomorphic. Now every twisted Lubin-Tate formal group law is strictly isomorphic to one of the form $G_{\omega}(X, Y)=g_{\omega}^{-1}\left(g_{\omega}(X)+g_{\omega}(Y)\right)$ with $g_{\omega}(X)=X$ $+\omega^{-1} X^{q}+\omega^{-1} \sigma(\omega)^{-1} X^{q^{2}}+\omega^{-1} \sigma(\omega)^{-1} \sigma^{2}(\omega)^{-1} X^{q^{3}}+\cdots$ which permits us to concentrate on the case $F(X, Y)=G_{\omega}(X, Y)$ for some $\omega$. The formulas are more pleasing in this case, especially because the only constants which then appear are the $\sigma^{i}(\omega)$, which is esthetically attractive, because $\omega$ is an invariant of the strict isomorphism class of $F(X, Y)$.

The functors $W_{q, \infty}^{F}$ and the functor morphisms $E$ are Witt-vector-like and Artin-Hasse-exponential-like in that

(i) $W_{q, \infty}^{F}(B)=\left\{\left(b_{0}, b_{1}, \ldots\right) \mid b_{i} \in B\right\}$ as a set-valued functor and the $A$-algebra structure can be defined via suitable Witt-like polynomials $w_{q, n}^{F}\left(Z_{0}, \ldots, Z_{n}\right)$; cf. below for more details.

(ii) There exist a $\sigma$-semilinear $\boldsymbol{A}$-algebra homomorphism $\mathrm{f}$ (Frobenius) and a $\sigma^{-1}$-semilinear $A$-module homomorphism $\mathbf{V}$ (Verschiebung) with the expected properties, e.g. $\mathrm{fV}=\omega$ where $\omega$ is the uniformizing element of $A$ associated to $F$, and $\mathbf{f}(\mathbf{b}) \equiv \mathbf{b}^{q} \bmod \omega W_{q, \infty}^{F}(B)$.

(iii) If $k$, the residue field of $A$, is perfect and $l / k$ is a finite field extension, then $W_{q, \infty}^{F}(l)=B$, the ring of integers of the unique unramified extension $L / K$ which covers $l / k$.

(iv) The Artin-Hasse exponential $E$ is characterized by $w_{q, n}^{F} \circ E=f^{n}$ for all $n=0,1,2, \ldots$

I hope that these constructions will also be useful in a class-field theory setting. 
Meanwhile they have been important in formal $A$-module theory. The results in question have been announced in two notes, [9] and [10], and I now propose to take half a page or so to try to explain these results to some extent.

Let $R$ be a $\mathbf{Z}_{(p)}$-algebra and let $\operatorname{Cart}_{p}(R)$ be the Cartier-Dieudonné ring. This is a ring "generated" by two symbols $\mathrm{f}, \mathrm{V}$ over $W_{p, \infty}(R)$ subject to "the relations suggested by the notation used". For each formal group $F(X, Y)$ over $R$ let $C_{p}(F ; R)$ be its $\operatorname{Cart}_{p}(R)$ module of $p$-typical curves. Finally let $\hat{W}_{p, \infty}(-)$ be the formal completion of the functor $W_{p, \infty}(-)$. Then one has

(a) the functor $F \mapsto C_{p}(F ; R)$ is representable by $\hat{W}_{p, \infty}[3]$.

(b) The functor $F \mapsto C_{p}(F ; R)$ is an equivalence of categories between the category of formal groups over $R$ and a certain (explicitly describable) subcategory of $\operatorname{Cart}_{p}(R)$ modules [3].

(c) There exists a theory of "lifting" formal groups, in which the Artin-Hasse exponential $E: W_{p, \infty}(-) \rightarrow W_{p, \infty}\left(W_{p, \infty}(-)\right)$ plays an important rôle. These results relate to the so-called "Tapis de Cartier" and relate to certain conjectures of Grothendieck concerning crystalline cohomology ([4] and [5]).

Now let $A$ be a complete discrete valuation ring with residue field $k$ with $q$ elements (for simplicity and/or nontriviality of the theory). A formal $A$-module over $B \in \mathbf{A l g}_{A}$ is a formal group law $F(X, Y)$ over $B$ together with a ring homomorphism $\rho_{F}: A \rightarrow \operatorname{End}_{B}(F(X, Y))$, such that $\rho_{F}(a) \equiv a X \bmod ($ degree 2$)$. Then there exist complete analogues of (a), (b), (c) above for the category of formal $A$-modules over $B$. Here the rôle of $C_{p}(F ; R)$ is taken over by the $q$-typical curves $C_{q}(F ; B), W_{p, \infty}(-)$ and $\hat{W}_{p, \infty}$ are replaced by ramified-Witt-vector functors $W_{q, \infty}^{\pi}(-)$ and $\hat{W}_{q, \infty}^{\pi}(-)$ associated to an untwisted, i.e. $\sigma=$ id, Lubin-Tate formal group law over $A$ with associated uniformizing element $\pi$. Finally, the rôle of $E$ in (c) is taken over by the ramified Hasse-Witt exponential $W_{q, \infty}^{\pi}(-) \rightarrow$ $W_{q, \infty}^{\pi}\left(W_{q, \infty}^{\pi}(-)\right)$.

As we remarked in (i) above, it is perfectly possible to define and analyse $W_{q, \infty}^{F}(-)$ by starting with the polynomials $w_{q, n}^{F}(Z)$ and then proceeding along the lines of Witt's original paper. And, in fact, in the untwisted case, where $k$ is a field of $q$-elements, this has been done, independently of this paper, and independently of each other by E. Ditters [7], V. Drinfel'd [8], J. Casey (unpublished) and, very possibly, several others. In this case the relevant polynomials are of course the polynomials $X_{0}^{q^{n}}+\pi X_{1}^{q^{n-1}}+\cdots+\pi^{n-1} X_{n-1}^{q}+\pi^{n} X_{n}$.

Of course the twisted version is necessary if one wants to describe also all ramified discrete valuation rings with not necessarily finite residue fields. A second main reason for considering "twisted formal $A$-modules" is that there exist no nontrivial formal $A$-modules if the residue field of $A$ is infinite.

Let me add that, in my opinion, the formal group law approach to (ramified) Witt-vectors is technically and conceptually easier. Witness, e.g. the proof of Theorem 6.6 and the ease with which one defines Artin-Hasse exponentials in this setting (cf. $\$ \$ 6.1$ and 6.5 below). Also this approach removes some of the mystery and exclusive status of the particular Witt polynomials $X_{0}^{p^{n}}+$ $p X_{1}^{p^{n-1}}+\cdots+p^{n} X_{n}$ (unramified case), $X_{0}^{q^{n}}+\pi X_{1}^{q^{n-1}}+\cdots+\pi^{n} X_{n}$ (untwisted ramified case), 


$$
X_{0}^{q^{n}}+\sigma^{n-1}(\omega) X_{1}^{q^{n-1}}+\sigma^{n-1}(\omega) \sigma^{n-2}(\omega) X_{2}^{q^{n-2}}+\cdots+\sigma^{n-1}(\omega) \cdots \sigma(\omega) \omega X_{n}
$$

(twisted ramified case). From the theoretical (if not the esthetic and/or computational) point of view all polynomials $\tilde{w}_{q, n}\left(X_{0}, \ldots, X_{n}\right)=a_{n}^{-1}\left(a_{n} X_{0}^{q^{n}}+a_{n-1} X_{1}^{q^{n-1}}\right.$ $\left.+\cdots+a_{0} X_{n}\right) \in A[X]$ are equally good, provided $a_{0}=1, a_{2}, a_{3}, \ldots$ is a sequence of elements of $K$ such that $a_{i}-\omega^{-1} \sigma\left(a_{i-1}\right) \in A$ for all $i=1,2, \ldots$ (cf. in this connection also [6]).

\section{The functional-equation-integrality lemma.}

2.1. The setting. Let $A$ be a discrete valuation ring with maximal ideal $\mathrm{m}$, residue field $k$ of characteristic $p>0$ and field of quotients $K$. Both characteristic zero and characteristic $p>0$ are allowed for $K$. We use $v$ to denote the normalized exponential valuation on $K$ and $\omega$ always denotes a uniformizing element, i.e. $v(\omega)=1$ and $m=\omega A$. We assume that there exist a power $q$ of $p$ and an automorphism $\sigma$ of $K$ such that

$$
\sigma(\mathfrak{m})=\mathfrak{m}, \quad \sigma a \equiv a^{q} \bmod \mathfrak{m} \text { for all } a \in A .
$$

The ring $A$ does not need to be complete.

Further let $B \in \mathbf{A l g} \mathbf{g}_{A}$, the category of $A$-algebras. We suppose that $B$ is $A$-torsion free (i.e. that the natural map $B \rightarrow B \otimes_{A} K$ is injective) and we suppose that there exists an endomorphism $\tau: B \otimes_{A} K \rightarrow B \otimes_{A} K$ such that

$$
\tau(b) \equiv b^{q} \bmod \mathrm{m} B \quad \text { for all } b \in B \text {. }
$$

Finally let $f(X)$ be any power series over $B \otimes_{A} K$ of the form

$$
f(X)=b_{1} X+b_{2} X^{2}+\cdots, \quad b_{i} \in B, b_{1} \text { a unit of } B
$$

for which there exists a uniformizing element $\omega \in A$ such that

$$
f(X)-\omega^{-1} \tau_{*} f\left(X^{q}\right) \in B[[X]]
$$

where $\tau_{*}$ means "apply $\tau$ to the coefficients". In terms of the coefficients $b_{i}$ of $f(X)$ condition (2.5) means that

$$
\begin{aligned}
& b_{i} \in B \quad \text { if } q \text { does not divide } i \\
& b_{q i}-\omega^{-1} \tau\left(b_{i}\right) \in B \text { for all } i=1,2, \ldots
\end{aligned}
$$

2.7. Functional eQuAtion Lemma. Let $A, B, \sigma, \tau, K, p, q, f(X), \omega$ be as in 2.1 above such that (2.2.)-(2.6) hold. Then we have

(i) $F(X, Y)=f^{-1}(f(X)+f(Y))$ has its coefficients in $B$ and hence is a commutative one-dimensional formal group law over B. $\left(\right.$ Here $f^{-1}(X)$ is the "inverse function" power series of $f(X)$; i.e. $f^{-1}(f(X))=X$.)

(ii) If $g(X) \in B[[X]], g(0)=0$ and $h(X)=f(g(X))$ then we have $h(X)-$ $\omega^{-1} \tau_{*} h\left(X^{q}\right) \in B[[X]]$.

(iii) If $h(X) \in B \otimes_{A} K[[X]], h(0)=0$ and $h(X)-\omega^{-1} \tau_{*} h\left(X^{q}\right) \in B[[X]]$, then $f^{-1}(h(X)) \in B[[X]]$.

(iv) If $\alpha(X) \in B[[X]], \beta(X) \in B \otimes_{A} K[[X]], \alpha(0)=\beta(0)=0$ and $r, m \in N=$ $\{1,2, \ldots\}$, then $\alpha(X) \equiv \beta(X) \bmod \left(\omega^{\prime} B\right.$, degree $\left.m\right) \Leftrightarrow f(\alpha(X)) \equiv f(\beta(X))$ $\bmod \left(\omega^{r} B\right.$, degree $\left.m\right)$. 
Proof. This lemma is a quite special case of the functional equation lemmas of [11, cf. $\S \S 2.2$ and 10.2]. There are also infinite-dimensional versions. Here is a quick proof. First notice that (2.6) implies (with induction) that

$$
b_{j} \in \omega^{-i} B \text { if } j \text { is not divisible by } q^{i+1} \text {. }
$$

We now first prove a more general form of (ii). Let $g(Z)=g\left(Z_{1}, \ldots, Z_{m}\right) \in$ $B\left[\left[Z_{1}, \ldots, Z_{m}\right]\right], g(0)=0$. Then by the hypotheses of 2.1 we have

$$
g\left(Z_{1}, \ldots, Z_{m}\right)^{q^{\prime} n} \equiv \tau_{*} g\left(Z_{1}^{q}, \ldots, Z_{m}^{q}\right)^{q^{r-1} n} \bmod \left(\omega^{r} B\right) .
$$

Combining (2.8) and (2.9) and using (2.6) we see that $\bmod (B[[X]])$ we have

$$
\begin{aligned}
h(Z) & =f(g(Z))=\sum_{i=1}^{\infty} b_{i} g(Z)^{i} \equiv \sum_{j=1}^{\infty} b_{q j} g(Z)^{q j} \equiv \omega^{-1} \sum_{j=1}^{\infty} \tau\left(b_{j}\right) g(Z)^{q j} \\
& \equiv \omega^{-1} \sum_{j=1}^{\infty} \tau\left(b_{j}\right) \tau_{*} g\left(Z^{q}\right)^{j}=\omega^{-1} \tau_{*} f\left(\tau_{*} g\left(Z^{q}\right)\right)=\omega^{-1} \tau_{*} h\left(Z^{q}\right) .
\end{aligned}
$$

This proves (ii). To prove (i) we write $F(X, Y)=F_{1}(X, Y)+F_{2}(X, Y)+\cdots$, where $F_{n}(X, Y)$ is homogeneous of degree $n$. We now prove by induction that $F_{n}(X, Y) \in B[X, Y]$ for all $n=1,2, \ldots$ The induction starts because $F_{1}(X, Y)$ $=X+Y$. Now assume that $F_{1}(X, Y), \ldots, F_{m}(X, Y) \in B[X, Y]$. We know that $f(F(X, Y)) \equiv b_{1} F_{m+1}(X, Y)+f(g(X, Y)) \bmod ($ degree $m+2)$, where $g(X, Y)=$ $F_{1}(X, Y)+\cdots+F_{m}(X, Y)$. Hence, using the more general form of (ii) proved just above, we find $\bmod (B[[X, Y]]$, degree $m+2)$ :

$$
\begin{aligned}
f(F(X, Y)) & \equiv b_{1} F_{m+1}(X, Y)+f(g(X, Y)) \\
& \equiv b_{1} F_{m+1}(X, Y)+\omega^{-1} \tau_{*} f\left(\tau_{*} g\left(X^{q}, Y^{q}\right)\right) \\
& \equiv b_{1} F_{m+1}(X, Y)+\omega^{-1} \tau_{*} f\left(\tau_{*} F\left(X^{q}, Y^{q}\right)\right) \\
& =b_{1} F_{m+1}(X, Y)+\omega^{-1} \tau_{*} f\left(X^{q}\right)+\omega^{-1} \tau_{*} f\left(Y^{q}\right) \\
& \equiv b_{1} F_{m+1}(X, Y)+f(X)+f(Y)=b_{1} F_{m+1}(X, Y)+f(F(X, Y))
\end{aligned}
$$

where we have used the defining relation $f(F(X, Y))=f(X)+f(Y)$, which implies $\tau_{*} f\left(\tau_{*} F\left(X^{q}, Y^{q}\right)\right)=\tau_{*} f\left(X^{q}\right)+\tau_{*} f\left(Y^{q}\right)$, and where we have also used the fact that $F(X, Y) \equiv g(X, Y) \bmod ($ degree $m+1) \Rightarrow F\left(X^{q}, Y^{q}\right) \equiv g\left(X^{q}, Y^{q}\right) \bmod ($ degree $m$ $+2)$. It follows that $b_{1} F_{m+1}(X, Y) \equiv 0 \bmod (B[[X, Y]]$, degree $m+2)$ and hence $F_{m+1}(X, Y) \in B[X, Y]$ because $b_{1}$ is a unit.

The proof of (iii) is completely analogous to the proof of (i).

The implication $\Rightarrow$ of (iv) is easy to prove. If $\alpha(X) \equiv \beta(X) \bmod \left(\omega^{r} B\right.$, degree $m$ ) and $\alpha(X) \in B[[X]]$ then $\alpha(X)^{q^{i j}} \equiv \beta(X)^{q^{i j}} \bmod \left(\omega^{r+i} B\right.$, degree $\left.m\right)$ which, combined with (2.8), proves that $f(\alpha(X)) \equiv f(\beta(X)) \bmod \left(\omega^{r} B\right.$, degree $\left.m\right)$. To prove the inverse implication $\Leftarrow$ of (iv) we first do the special case

$$
f(\beta(X)) \equiv 0 \bmod \left(\omega^{r} B \text {, degree } m\right) \Rightarrow \beta(X) \equiv 0 \bmod \left(\omega^{r} B \text {, degree } m\right) .
$$

Now $\beta(X) \equiv 0 \bmod ($ degree 1$)$, hence $f(\beta(X))=b_{1} \beta(X)+b_{2} \beta(X)^{2}+\cdots \equiv 0$ $\bmod \left(\omega^{r} B, \operatorname{degree} m\right)$, implies $\beta(X) \equiv 0 \bmod \left(\omega^{r} B\right.$, degree 2$)$, if $m>2$ (if $m=1$ there is nothing to prove), because $b_{1}$ is a unit. Now assume with induction that 
$\beta(X) \equiv 0 \bmod \left(\omega^{r} B\right.$, degree $\left.n\right)$ for some $n<m$. Then, because $\beta(X) \equiv$ $0 \bmod ($ degree 1$)$ we have $\beta(X)^{i} \equiv 0 \bmod \left(\omega^{r i} B, \operatorname{degree}(n+i-1)\right)$ and hence $b_{j} \beta(X)^{j} \equiv 0 \bmod \left(\omega^{r} B\right.$, degree $\left.n+1\right)$ if $j>2$. Hence $f(\beta(X)) \equiv$ $0 \bmod \left(\omega^{r} B\right.$, degree $\left.m\right)$ then gives $b_{1} \beta(X) \equiv 0 \bmod \left(\omega^{r} B\right.$, degree $\left.n+1\right)$, so that $\beta(X) \equiv 0 \bmod \left(\omega^{r} B\right.$, degree $\left.n+1\right)$ because $b_{1}$ is a unit. This proves this special case of (iv). Now let $f(\alpha(X)) \equiv f(\beta(X)) \bmod \left(\omega^{r} B\right.$, degree $\left.m\right)$. Write $\gamma(X)=$ $f(\beta(X))-f(\alpha(X))$ and $\delta(X)=f^{-1}(\gamma(X))$. Then $\delta(X) \equiv 0 \bmod \left(\omega^{r} B\right.$, degree $\left.m\right)$ by the special case just proved, and $\beta(X)=f^{-1}(f(\alpha(X))+f(\delta(X)))=F(\alpha(X), \delta(X))$ $\equiv \alpha(X) \bmod \left(\omega^{r} B\right.$, degree $\left.m\right)$ because $F(X, Y)$ has integral coefficients, $F(X, 0)=0$ and because $\alpha(X)$ is integral. This concludes the proof of the Functional Equation Lemma 2.7.

\section{Twisted Lubin-Tate formal $\boldsymbol{A}$-modules.}

3.1. Construction and definition. Let $A, K, k, p, \mathfrak{m}, \sigma, q$ be as in 2.1 above. We consider a power series $f(X)=X+c_{2} X^{2}+\cdots \in K[[X]]$ such that there exists a uniformizing element $\omega \in \mathfrak{m}$ such that

$$
f(X)-\omega^{-1} \sigma_{*} f\left(X^{q}\right) \in A[[X]] .
$$

There are many such power series. The simplest are obtained as follows. Choose a uniformizing element $\omega$ of $A$. Define

$$
g_{\omega}(X)=X+\omega^{-1} X^{q}+\omega^{-1} \sigma(\omega)^{-1} X^{q^{2}}+\omega^{-1} \sigma(\omega)^{-1} \sigma^{2}(\omega)^{-1} X^{q^{3}}+\cdots
$$

Given such a power series $f(X)$, part (i) of the Functional Equation Lemma says that

$$
F(X, Y)=f^{-1}(f(X)+f(Y))
$$

has its coefficients in $A$, and hence is a one-dimensional formal group law over $A$. We shall call the formal group laws thus obtained twisted Lubin-Tate formal $A$-modules over $A$. The twisted Lubin-Tate formal $A$-module is called $q$-typical if the power series $f(X)$ that it is obtained from is of the form

$$
f(X)=X+a_{1} X^{q}+a_{2} X^{q^{2}}+\cdots \cdot
$$

From now on all twisted Lubin-Tate formal $A$-modules will be assumed to be $q$-typical. This is hardly a restriction because of Lemma 3.6 below.

3.6. Lemma. Let $f(X)=X+c_{2} X^{2}+\cdots \in K[[X]]$ be such that (3.2) holds. Let $\hat{f}(X)=\sum_{i=0}^{\infty} a_{i} X^{q^{i}}$ with $a_{0}=1, a_{i}=c_{q}$. Then $u(X)=\hat{f}^{-1}(f(X)) \in A[[X]]$ so that $F(X, Y)$ and $\hat{F}(X, Y)$ are strictly isomorphic formal group laws over $A$.

Proof. It follows from the definition of $\hat{f}(X)$, that $\hat{f}(X)$ also satisfies (3.2). The integrality of $u(X)$ now follows from part (iii) of the Functional Equation Lemma.

3.7. Remarks. Let $k$, the residue field of $K$, be finite with $q$ elements, and let $\sigma=$ id. Then the twisted Lubin-Tate formal $A$-modules over $A$ as defined above are precisely the Lubin-Tate formal group laws defined in [12], i.e. they are precisely the formal $A$-modules of $A$-height 1 . If $k$ is infinite there exist no nontrivial formal $A$-modules (cf. [11, Corollary 21.4.23]). This is a main reason for also considering twisted Lubin-Tate formal group laws. 
3.8. REMARK. Let $f(X) \in K[[X]]$ be such that (3.2) holds for a certain uniformizing element $\omega$. Then $\omega$ is uniquely determined by $f(X)$, because $a_{i}-\omega^{-1} \sigma\left(a_{i-1}\right) \in$ $A \Rightarrow \omega \equiv a_{i}^{-1} \sigma\left(a_{i-1}\right) \bmod \omega^{2 i} A$ as $v\left(a_{i}\right)=-i$. Using parts (ii) and (iii) of the Functional Equation Lemma we see that $\omega$ is in fact an invariant of the strict isomorphism class of $F(X, Y)$. Inversely, given $\omega$ we can construct $g_{\omega}(X)$ as in (3.3) and then $g_{\omega}^{-1}(f(X))=u(X)$ is integral so that $F(X, Y)$ and $G_{\omega}(X, Y)=$ $g_{\omega}^{-1}\left(g_{\omega}(X)+g_{\omega}(Y)\right)$ are strictly isomorphic formal group laws. In case \#k=q and $\sigma=\mathrm{id}, \omega$ is in fact an invariant of the isomorphism class of $F(X, Y)$. For some more results on isomorphisms and endomorphisms of twisted Lubin-Tate formal $A$-modules cf. [11], especially $\S \S 8.3,20.1,21.8,24.5$.

4. Curves and $q$-typical curves. Let $F(X, Y)$ be a $q$-typical twisted Lubin-Tate formal $A$-module obtained via (3.4) from a power series $f(X)=X+a_{1} X^{q}+a_{2} X^{q^{2}}$ $+\cdots$.

4.1. Curves. Let $\mathbf{A l g}_{A}$ be the category of $A$-algebras. Let $B \in \mathbf{A l g}_{A}$. A curve in $F$ over $B$ is simply a power series $\gamma(t) \in B[[t]]$ such that $\gamma(0)=0$. Two curves can be added by the formula $\gamma_{1}(t)+{ }_{F} \gamma_{2}(t)=F\left(\gamma_{1}(t), \gamma_{2}(t)\right)$, giving us an abelian group $C(F ; B)$. Further, if $\phi: B_{1} \rightarrow B_{2}$ is in $\mathbf{A l g}_{A}$, then $\gamma(t) \mapsto \phi_{*} \gamma(t)$ (= "apply $\phi$ to the coefficients") defines a homomorphism of abelian groups $C\left(F ; B_{1}\right) \rightarrow C\left(F ; B_{2}\right)$. This defines an abelian-group-valued functor $C(F ;-): \mathbf{A l g}_{A} \rightarrow \mathbf{A b}$. There is a natural filtration on $C(F ;-)$ defined by the filtration subgroups $C^{n}(F ; B)=\{\gamma(t)$ $\in C(F ; B) \mid \gamma(t) \equiv 0 \bmod ($ degree $n)\}$. The groups $C(F ; B)$ are complete with respect to the topology defined by the filtration $C^{n}(F ; B), n=1,2, \ldots$.

The functor $C(F ;-)$ is representable by the $A$-algebra $A[S]=A\left[S_{1}, S_{2}, \ldots\right]$. The isomorphism $\operatorname{Alg}_{A}(A[S], B) \stackrel{\sim}{\rightarrow} C(F ; B)$ is given by

$$
\phi \mapsto \sum_{i=1}^{\infty} F\left(S_{i}\right) t^{i}
$$

i.e. by $\phi \mapsto \phi_{*} \gamma_{S}(t)$, where $\gamma_{S}(t)$ is the "universal curve"

$$
\gamma_{S}(t)=\sum_{i=1}^{\infty} F S_{i} t^{i} \in C(F ; A[S]) .
$$

Here the superscript $F$ means that we sum in the group $C(F ; B)$ just defined (to avoid possible confusion with ordinary sums).

4.2. q-typification. Let $\gamma_{S}(t) \in C(F ; A[S])$ be the universal curve. Consider the power series

$$
h(t)=f\left(\gamma_{S}(t)\right)=\sum_{i=1}^{\infty} x_{i}(S) t^{i}
$$

Let $\tau: K[S] \rightarrow K[S]$ be the ring endomorphism defined by $\tau(a)=\sigma(a)$ for $a \in K$ and $\tau\left(S_{i}\right)=S_{i}^{q}$ for $i=1,2, \ldots$ Then the hypotheses of 2.1 are fulfilled and it follows from part (ii) of the Functional Equation Lemma that $h(t)-\omega^{-1} \tau_{*} h\left(t^{q}\right) \in$ $A[S][[t]]$. Now let $\hat{h}(t)=\sum_{i=0}^{\infty} x_{q^{i}}(S) t^{q^{i}}$. Then, obviously, also $\hat{h}(t)-\omega^{-1} \tau_{*} \hat{h}\left(t^{q}\right) \in$ $A[S][[t]]$ and by part (iii) of the Functional Equation Lemma it follows that 


$$
\varepsilon_{q} \gamma_{S}(t)=f^{-1}\left(\sum_{i=0}^{\infty} x_{q^{i}}(S) t^{q^{i}}\right)
$$

is an element of $A[S][[t]]$. We now define a functorial group homomorphism $\varepsilon_{q}$ : $C(F ;-) \rightarrow C(F ;-)$ by the formula

$$
\varepsilon_{q} \gamma(t)=\left(\phi_{\gamma}\right)_{*}\left(\varepsilon_{q} \gamma_{S}(t)\right)
$$

for $\gamma(t) \in C(F ; B)$, where $\phi_{\gamma}: A[S] \rightarrow B$ is the unique $A$-algebra homomorphism such that $\left(\phi_{\gamma}\right)_{*} \gamma_{S}(t)=\gamma(t)$.

4.5. Lemma. Let $B$ be $A$-torsion free so that $B \rightarrow B \otimes_{A} K$ is injective. Then we have for all $\gamma(t) \in C(F ; B)$,

$$
f(\gamma(t))=\sum_{i=1}^{\infty} b_{i} t^{i} \Rightarrow f\left(\varepsilon_{q} \gamma(t)\right)=\sum_{j=0}^{\infty} b_{q} t^{q^{\prime}}
$$

and $\varepsilon_{q} C(F ; B)=\left\{\gamma(t) \in C(F ; B) \mid f(\gamma(t))=\Sigma c_{j} t^{q^{\prime}}\right.$ for certain $\left.c_{j} \in B \otimes_{A} K\right\}$.

Proof. Immediate from (4.3) and (4.4).

4.7. LEMMA. $\varepsilon_{q}$ is a functorial, idempotent, group endomorphism of $C(F ;-)$.

Proof. $\varepsilon_{q}$ is functorial by definition. The facts that $\varepsilon_{q} \varepsilon_{q}=\varepsilon_{q}$ and that $\varepsilon_{q}$ is a group homomorphism are obvious from Lemma 4.5 in case $B$ is $A$-torsion free. Functoriality then implies that these properties hold for all $A$-algebras $B$.

4.8. The functor $C_{q}(F ;-)$ of q-typical curves. We now define the abelian-groupvalued functor $C_{q}(F ;-)$ as

$$
C_{q}(F ;-)=\varepsilon_{q} C(F ;-) \text {. }
$$

For each $n \in \mathbf{N} \cup\{0\}$ let $C_{q}^{(n)}(F ; B)$ be the subgroup $C_{q}(F ; B) \cap C^{q^{n}}(F ; B)$. These groups define a filtration on $C_{q}(F ; B)$, and $C_{q}(F ; B)$ is complete with respect to the topology defined by this filtration.

The functor $C_{q}(F ;-)$ is representable by the $A$-algebra $A[T]=A\left[T_{0}, T_{1}, \ldots\right]$. Indeed, writing $f(X)=\sum_{i=0}^{\infty} a_{i} X^{q^{i}}$ we have

$$
f\left(\gamma_{S}(t)\right)=f\left(\sum_{i=1}^{\infty}{ }^{F} S_{i} t^{i}\right)=\sum_{j=0}^{\infty} \sum_{i=1}^{\infty} a_{j} S_{i}^{q^{\prime} t^{q^{i}}}
$$

and it follows that

$$
\varepsilon_{q} \gamma_{S}(t)=\sum_{j=0}^{\infty} F S_{q} t^{q^{j}}
$$

From this one easily obtains that the functor $C_{q}(F ;-)$ is representable by $A[T]$. The isomorphism $\operatorname{Alg}_{A}(A[T], B) \stackrel{\sim}{\rightarrow} C_{q}(F ; B)$ is given by

$$
\phi \mapsto \sum_{i=0}^{\infty} F\left(T_{i}\right) t^{q^{\prime}}=\phi_{*}\left(\gamma_{T}(t)\right),
$$

where $\gamma_{T}(t)$ is the universal $q$-typical curve

$$
\gamma_{T}(t)=\sum_{i=0}^{\infty}{ }^{F} T_{i} t^{q^{\prime}} \in C_{q}(F ; A[T])
$$


4.11. Remarks. The explicit formulas of 4.8 above depend on the fact that $F$ was supposed to be $q$-typical. In general slightly more complicated formulae hold. For arbitrary formal groups $q$-typification (i.e. $\varepsilon_{q}$ ) is not defined (unless $q=p$ ). But a similar notion of $q$-typification exists for formal $A$-modules of any height and any dimension if \#k=q.

5. The $A$-algebra structure on $C_{q}(F ;-)$, Frobenius and Verschiebung.

5.1. From now on we assume that $f(X)=g_{\omega}(X)=X+\omega^{-1} X^{q}+\omega^{-1} \sigma(\omega)^{-1} X^{q^{2}}$ $+\cdots$ for a certain uniformizing element $\omega$. Otherwise we keep the notations and assumptions of $\S 4$. Thus we now have $a_{i}^{-1}=\omega \sigma(\omega) \ldots \sigma^{i-1}(\omega), a_{0}=1$. This restriction to "logarithms" $f(X)$ of the form $g_{\omega}(X)$ is not very serious, because every twisted Lubin-Tate formal $A$-module over $A$ is strictly isomorphic to a $G_{\omega}(X, Y)$, (cf. Remark 3.8), and one can use the strict isomorphism $g_{\omega}^{-1}(f(X))$ to transport all the extra structure on $C_{q}(F ;-)$ which we shall define in this section. The restriction $f(X)=g_{\omega}(X)$ does have the advantage of simplifying the defining formulas (5.4), (5.5), (5.8), . . somewhat, and it makes them look rather more natural especially in view of the fact that $\omega$, the only "constant" which appears, is an invariant of strict isomorphism classes of twisted Lubin-Tate formal $\boldsymbol{A}$-modules (cf. Remark 3.8 above).

In this section we shall define an $A$-algebra structure on the functor $C_{q}(F ;-)$ and two endomorphisms $f_{\omega}$ and $V_{q}$. These constructions all follow the same pattern, the same pattern as was used to define and analyse $\varepsilon_{q}$ in $\$ 4$ above. First one defines the desired operations for universal curves like $\gamma_{T}(t)$ which are defined over rings like $A[T]$, which, and this is the crucial point, admit an endomorphism $\tau: K[T] \rightarrow K[T]$, viz. $\tau(a)=\sigma(a), \tau\left(T_{i}\right)=T_{i}^{q}$, which extends $\sigma$ and which is such that $\tau(x) \equiv x^{q} \bmod \omega A[T]$. In such a setting the Functional Equation Lemma assures us that our constructions do not take us out of $C(F ;-)$ or $C_{q}(F ;-)$. Second, the definitions are extended via representability and functoriality, and thirdly, one derives a characterization which holds over $\boldsymbol{A}$-torsion free rings, and using this, one proves the various desired properties like associativity of products, $\sigma$-semilinearity of $\mathbf{f}_{\omega}$, etc.

5.2. Constructions. Let $\gamma_{T}(t)$ be the universal $q$-typical curve (4.10). We write

$$
f\left(\gamma_{T}(t)\right)=\sum_{i=0}^{\infty} x_{i}(T) t^{q^{i}} .
$$

Let $f(X)=g_{\omega}(X)=\sum_{i=0}^{\infty} a_{i} X^{q^{i}}$, i.e. $a_{i}=\omega^{-1} \sigma(\omega)^{-1} \ldots \sigma^{i-1}(\omega)^{-1}$ and let $a \in A$.

We define

$$
\begin{aligned}
& \{a\}_{F} \gamma_{T}(t)=f^{-1}\left(\sum_{i=0}^{\infty} \sigma^{i}(a) x_{i}(T) t^{q^{i}}\right), \\
& \mathbf{f}_{\omega} \gamma_{T}(t)=f^{-1}\left(\sum_{i=0}^{\infty} \sigma^{i}(\omega) x_{i+1}(T) t^{q^{i}}\right) .
\end{aligned}
$$

The Functional Equation Lemma now assures us that (5.4) and (5.5) define elements of $C(F ; A[T])$, which then are in $C_{q}(F ; A[T])$ by Lemma 4.5. To illustrate 
this we check the hypotheses necessary to apply (iii) of 2.7 in the case of $\mathbf{f}_{\boldsymbol{\omega}}$. Let $\tau$ : $K[T] \rightarrow K[T]$ be as in 5.1 above. Then by part (ii) of the Functional Equation Lemma we know that

$$
x_{0} \in A[T], \quad x_{i+1}-\omega^{-1} \tau\left(x_{i}\right)=c_{i} \in A[T] .
$$

It follows by induction that

$$
x_{i} \in \omega^{-i} A[T]
$$

and we also know that

$$
v\left(a_{i}^{-1}\right)=v\left(\omega \sigma(\omega) \ldots \sigma^{i-1}(\omega)\right)=i
$$

where $v$ is the normalized exponential valuation on $K$. We thus have $\sigma^{0}(\omega) x_{1}=\omega x_{1}$ $\in A[T]$ and

$$
\begin{aligned}
\sigma^{i}(\omega) x_{i+1}-\omega^{-1} \tau\left(\sigma^{i-1}(\omega) x_{i}\right) & =\sigma^{i}(\omega) c_{i}+\sigma^{i}(\omega) \omega^{-1} \tau\left(x_{i}\right)-\omega^{-1} \tau\left(\sigma^{i-1}(\omega) x_{i}\right) \\
& =\sigma^{i}(\omega) c_{i} \in A[T]
\end{aligned}
$$

Hence part (iii) of the Functional Equation Lemma says that $\mathbf{f}_{\omega} \gamma_{T}(t) \in$ $C(F ; A[T])$.

To define the multiplication on $C_{q}(F ;-)$ we need two independent universal $q$-typical curves. Let $\gamma_{T}(t)=\Sigma^{F} T_{i} t^{q^{\prime}}, \delta_{\hat{T}}(t)=\Sigma^{F} \hat{T}_{i} t^{q^{\prime}} \in C_{q}(F ; A[T ; \hat{T}])$. We define

$$
\gamma_{T}(t) * \delta_{\hat{T}}(t)=f^{-1}\left(\sum_{i=0}^{\infty} a_{i}^{-1} x_{i} y_{i} t^{q^{\prime}}\right)
$$

where $f\left(\gamma_{T}(t)\right)=\sum x_{i} t^{q^{i}}, f\left(\delta_{\hat{T}}(t)\right)=\sum y_{i} t^{q^{i}}$. To prove that (5.8) defines something integral we proceed as usual. We have $x_{0}, y_{0} \in A[T ; \hat{T}], x_{i+1}-\omega^{-1} \tau\left(x_{i}\right)=c_{i} \in$ $A[T ; \hat{T}], y_{i+1}-\omega^{-1} \tau\left(y_{i}\right)=d_{i} \in A[T ; \hat{T}]$, where $\tau: K[T ; \hat{T}] \rightarrow K[T ; \hat{T}]$ is defined by $\tau(a)=\sigma(a)$ for $a \in K$, and $\tau\left(T_{i}\right)=T_{i}^{q}, \tau\left(\hat{T}_{i}\right)=T_{i}^{q}, i=0,1,2, \ldots$ Then $a_{0} x_{0} y_{0}=x_{0} y_{0} \in A[T ; \hat{T}]$ and

$$
\begin{aligned}
a_{i+1}^{-1} x_{i+1} y_{i+1} & -\omega^{-1} \tau\left(a_{i}^{-1} x_{i} y_{i}\right) \\
& =\omega \sigma\left(a_{i}\right)^{-1}\left(c_{i}+\omega^{-1} \tau\left(x_{i}\right)\right)\left(d_{i}+\omega^{-1} \tau\left(y_{i}\right)\right)-\omega^{-1} \sigma\left(a_{i}^{-1}\right) \tau\left(x_{i}\right) \tau\left(y_{i}\right) \\
& =\omega \sigma\left(a_{i}^{-1}\right) c_{i} d_{i}+\sigma\left(a_{i}\right)^{-1}\left(c_{i} \tau\left(y_{i}\right)+d_{i} \tau\left(x_{i}\right)\right) \in A[T ; \hat{T}]
\end{aligned}
$$

by (5.6) and (5.7).

5.9. Definition. Let $\gamma(t), \delta(t)$ be two $q$-typical curves in $F$ over $B \in \mathbf{A l g}_{\boldsymbol{A}}$. Let $\phi$ : $A[T] \rightarrow B$ be the unique $A$-algebra homomorphism such that $\phi_{*} \gamma_{T}(t)=\gamma(t)$, and let $\psi: A[T ; \hat{T}] \rightarrow B$ be the unique $A$-algebra homomorphism such that $\psi_{*} \gamma_{T}(t)=$ $\gamma(t), \psi_{*} \delta_{\hat{T}}(t)=\delta(t)$. Let $a \in A$. We define

$$
\begin{aligned}
\{a\}_{F} \gamma(t) & =\phi_{*}\left(\{a\}_{F} \gamma_{T}(t)\right), \\
\mathbf{f}_{\omega} \gamma(t) & =\phi_{*}\left(\mathbf{f}_{\omega} \gamma_{T}(t)\right), \\
\gamma(t) * \delta(t) & =\psi_{*}\left(\gamma_{T}(t) * \delta_{T}(t)\right)
\end{aligned}
$$

5.13. Characterizations. Let $B$ be an $A$-torsion free $A$-algebra; i.e. $B \rightarrow B \otimes_{A} K$ is injective, then the definitions (5.10)-(5.12) are characterized by the implications 


$$
\begin{gathered}
f(\gamma(t))=\sum_{i=0}^{\infty} x_{i} t^{q^{i}} \Rightarrow f\left(\{a\}_{F} \gamma(t)\right)=\sum_{i=0}^{\infty} \sigma^{i}(a) x_{i} t^{q^{i}} \\
f(\gamma(t))=\sum_{i=0}^{\infty} x_{i} t^{q^{i}} \Rightarrow f\left(f_{\omega} \gamma(t)\right)=\sum_{i=0}^{\infty} \sigma^{i}(\omega) x_{i+1} t^{q^{i}} \\
f(\gamma(t))=\sum_{i=0}^{\infty} x_{i} t^{q^{i}}, \quad f(\delta(t))=\sum_{i=0}^{\infty} y_{i} t^{q^{i}} \Rightarrow \\
f(\gamma(t) * \delta(t))=\sum_{i=0}^{\infty} a_{i}^{-1} x_{i} y_{i} t^{q^{i}} .
\end{gathered}
$$

This follows immediately from (5.4), (5.5) (5.8) compared with (5.10)-(5.12), because $\phi_{*}$ and $\psi_{*}$ are defined by applying $\phi$ and $\psi$ to coefficients, and because $\gamma(t) \mapsto f(\gamma(t))$ is injective, if $B$ is $A$-torsion free.

5.17. Theorem. The operators $\{a\}_{F}$ defined by (5.10) define a functorial A-module structure on $C_{q}(F ;-)$. The multiplication * defined by $(5.12)$ then makes $C_{q}(F ;-)$ an $A$-algebra-valued functor, with as unit element the q-typical curve $\gamma_{0}(t)=t$. The operator $\mathbf{f}_{\omega}$ is a $\sigma$-semilinear A-algebra homomorphism, i.e. $\mathbf{f}_{\omega}$ is a unit and multiplication-preserving group endomorphism such that $\mathbf{f}_{\omega}\{a\}_{F}=\{\sigma(a)\}_{F} f_{\omega}$.

Proof. In case $B$ is $A$-torsion free the various identities in $C_{q}(F ; B)$ like $\left(\{a\}_{F} \gamma(t)\right) * \delta(t)=\{a\}_{F}(\gamma(t) * \delta(t)), \quad \gamma(t) *\left(\delta(t)+_{F} \varepsilon(t)\right)=(\gamma(t) * \delta(t))$ $+_{F}(\gamma(t) * \varepsilon(t)), \ldots$ are obvious from the characterizations (5.14)-(5.16). The theorem then follows by functoriality.

5.18. Verschiebung. We now define the Verschiebung operator $\mathrm{V}_{q}$ on $C_{q}(F ;-)$ by the formula $\mathrm{V}_{q} \gamma(t)=\gamma\left(t^{q}\right)$. (It is obvious from Lemma 4.5 that this takes $q$-typical curves into $q$-typical curves.) In terms of the logarithm $f(X)$ one has for curves $\gamma(t)$ over $A$-torsion free $A$-algebras $B$,

$$
f(\gamma(t))=\sum_{i=0}^{\infty} x_{i} t^{q^{i}} \Rightarrow f\left(\mathbf{V}_{q} \gamma(t)\right)=\sum_{i=0}^{\infty} x_{i} t^{q^{i+1}}
$$

5.20. THEOREM. For $q$-typical curves $\gamma(t)$ in $F$ over an A-algebra $B$,

$$
\begin{gathered}
\mathbf{f}_{\omega} \mathbf{v}_{q} \gamma(t)=\{\omega\}_{F} \gamma(t), \\
\mathbf{f}_{\omega} \gamma(t) \equiv \gamma(t)^{* q} \bmod \{\omega\}_{F} C_{q}(F ; B) .
\end{gathered}
$$

Proof. (5.21) is immediate from (5.14), (5.15) and (5.19) in the case of $A$-torsion free $B$ and then follows in general by functoriality. The proof of (5.22) is a bit longer. It suffices to prove (5.22) for curves $\gamma(t) \in C_{q}(F ; A[T])$. In fact it suffices to prove (5.22) for $\gamma(t)=\gamma_{T}(t)$, the universal curve of (4.10). Let

$$
\delta(t)=f^{-1}\left(\sum_{i=0}^{\infty} y_{i} t^{q^{i}}\right), \quad y_{i}=x_{i+1}-\sigma^{i}(\omega)^{-1} a_{i} a_{i}^{-q} x_{i}^{q},
$$

where the $x_{i}, i=0,1,2, \ldots$, are determined by $f(\gamma(t))=\Sigma x_{i} t^{q^{i}}$. It then follows from (5.14)-(5.16) that indeed $f_{\omega} \gamma(t)-\gamma(t)^{* q}=\{\omega\}_{F} \delta(t)$, provided that we can show that $\delta(t)$ is integral, i.e. that $\delta(t) \in C_{q}(F ; A[T])$. To see this it suffices to show 
that $y_{0} \in A[T]$ and $y_{i+1}-\omega^{-1} \tau\left(y_{i}\right) \in A[T]$ because of part (iii) of the Functional Equation Lemma. Let $c_{i}=x_{i+1}-\omega^{-1} \tau\left(x_{i}\right) \in A[T]$. Then

$$
y_{0}=x_{1}-\sigma^{0}(\omega)^{-1} x_{0}^{q}=c_{0}+\omega^{-1} \tau\left(x_{0}\right)-\omega^{-1} x_{0}^{q} \in A[T]
$$

because $\tau\left(x_{0}\right) \equiv x_{0}^{q} \bmod \omega A[T]$. Further from $x_{i+1}=c_{i}+\omega^{-1} \tau\left(x_{i}\right)$ we find

$$
a_{i+1}^{-1} x_{i+1}=\omega \sigma(\omega) \ldots \sigma^{i}(\omega) c_{i}+\sigma(\omega) \ldots \sigma^{i}(\omega) \tau\left(x_{i}\right)=\omega^{i+1} d_{i}+\tau\left(a_{i}^{-1} x_{i}\right)
$$

for a certain $d_{i} \in A[T]$, and hence

$$
a_{i+1}^{-q} x_{i+1}^{q}=\tau\left(a_{i}^{-q} x_{i}^{q}\right)+\omega^{i+2} e_{i}
$$

for a certain $e_{i} \in A[T]$. It follows that

$$
\begin{aligned}
y_{i+1}-\omega^{-1} \tau\left(y_{i}\right)= & x_{i+2}-\sigma^{i+1}(\omega)^{-1} a_{i+1} a_{i+1}^{-q} x_{i+1}^{q}-\omega^{-1} \tau\left(x_{i+1}\right) \\
& +\omega^{-1} \tau\left(\sigma^{i}(\omega)^{-1} a_{i} a_{i}^{-q} x_{i}^{q}\right) \\
= & c_{i+1}-\sigma^{i+1}(\omega)^{-1}\left(a_{i+1} a_{i+1}^{-q} x_{i+1}^{q}-\omega^{-1} \sigma\left(a_{i}\right) \tau\left(a_{i}^{-q} x_{i}^{q}\right)\right) \\
= & c_{i+1}-\sigma^{i+1}(\omega)^{-1} a_{i+1}\left(a_{i+1}^{-q} x_{i+1}^{q}-\tau\left(a_{i}^{-q} x_{i}^{q}\right)\right) \in A[T]
\end{aligned}
$$

because $a_{i+1}=\omega^{-1} \sigma\left(a_{i}\right)$ and because of (5.24). (Recall that $v\left(a_{i+1}\right)=-i-1$ by (5.7).) This concludes the proof of Theorem 5.20.

6. Ramified Witt vectors and ramified Artin-Hasse exponentials. We keep the assumptions and notations of $\S 5$.

6.1. A preliminary Artin-Hasse exponential. Let $B$ be an $A$-algebra which is $A$-torsion free and which admits an endomorphism $\tau: B \otimes_{A} K \rightarrow B \otimes_{A} K$ which restricts to $\sigma$ on $A \otimes_{A} K=K \subset B \otimes_{A} K$ and which is such that $\tau(b) \equiv b^{q}$ $\bmod \omega B$. We define a map $\Delta_{B}: B \rightarrow C_{q}(F ; B)$ as follows.

$$
\Delta_{B}(b)=f^{-1}\left(\sum_{i=0}^{\infty} \tau^{i}(b) a_{i} t^{q^{i}}\right) .
$$

This is well defined by part (iii) of the Functional Equation Lemma. A quick check by means of (5.14)-(5.16) shows that $\Delta_{B}$ is a homomorphism of $\boldsymbol{A}$-algebras such that, moreover,

$$
\Delta_{B} \circ \tau=\mathbf{f}_{\omega} \circ \Delta_{B}
$$

(because $\left.\sigma^{i}(\omega) a_{i+1}=a_{i}\right)$, and that $\Delta_{B}$ is functorial in the sense that if $\left(B^{\prime}, \tau^{\prime}\right)$ is a second such $A$-algebra with endomorphism $\tau^{\prime}$ of $B^{\prime} \otimes_{A} K$ and $\phi: B \rightarrow B^{\prime}$ is an $A$-algebra homomorphism such that $\tau^{\prime} \phi=\phi \tau$, then $C_{q}(F ; \phi) \circ \Delta_{B}=\Delta_{B^{\prime}} \circ \phi$.

6.4. Remark. Using $(B, \tau)$ instead of $(A, \sigma)$ we can view $F(X, Y)$ as a twisted Lubin-Tate formal $B$-module over $B$, if we are willing to extend the definition a bit, because, of course, $B$ need not be a discrete valuation ring, nor is $B \otimes_{A} K$ necessarily the quotient field of $B$. In fact $B$ need not even be an integral domain. If we view $F(X, Y)$ in this way then $\Delta_{B}: B \rightarrow C_{q}(F ; B)$ is just the $B$-algebra structure map of $C_{q}(F ; B)$.

6.5. Now let $B$ be any $A$-algebra. Then $C_{q}(F ; B)$ is an $A$-algebra which admits an endomorphism $\tau$, viz. $\tau=\mathbf{f}_{\omega}$, which, as $\tau x \equiv x^{q} \bmod \omega$ by (5.22), satisfies the hypotheses of 6.1 above (because $f_{\omega}$ is $\sigma$-semilinear). It is also immediate from 
(5.10) and (5.4), cf. also (5.14), that $C_{q}(F ; B)$ is always $A$-torsion free. Substituting $C_{q}(F ; B)$ for $B$ in 6.1 we therefore find $A$-algebra homomorphisms $E_{B}: C_{q}(F ; B) \rightarrow$ $C_{q}\left(F ; C_{q}(F ; B)\right)$ which are functorial in $B$ because $\mathbf{f}_{\omega}$ is functorial, and because of the functoriality property of the $\Delta_{B}$ mentioned in 6.1 above. This functorial $A$-algebra homomorphism is in fact the ramified Artin-Hasse exponential we are seeking and, as is shown by the next theorem, $C_{q}(F ; B)$ is the desired ramifiedWitt-vector functor.

6.6. TheOREM. Let $A$ be complete with perfect residue field $k$. Let $B$ be the ring of integers in a finite unramified extension $L$ of $K$. Let $l$ be the residue field of $B$. Consider the composed map

$$
\mu_{B}: B \stackrel{\Delta_{B}}{\rightarrow} C_{q}(F ; B) \rightarrow C_{q}(F ; l) .
$$

Then $\mu_{B}$ is an isomorphism of $A$-algebras. Moreover if $\tau: B \rightarrow B$ is the unique extension of $\sigma: A \rightarrow A$ such that $\tau(b) \equiv b^{q} \bmod B$, then $\mathbf{f}_{\omega} \mu_{B}=\mu_{B} \tau$, i.e. $\tau$ and $\mathbf{f}_{\omega}$ correspond under $\mu_{B}$.

Proof. Let $b \in B$. Consider $\Delta_{B}\left(\omega^{r} b\right)$. Then from (6.2) we see that

$$
f\left(\Delta_{B}\left(\omega^{r} b\right)\right) \equiv a_{r} \tau^{r}\left(\omega^{r}\right) \tau^{r}(b) t^{q^{r}} \bmod \left(\omega B, \text { degree } q^{r+1}\right) .
$$

By part (iv) of the Functional Equation Lemma 2.7 it follows that

$$
\Delta_{B}\left(\omega^{r} b\right) \equiv y_{r} \tau^{r}(b) t^{q^{r}} \bmod \left(\omega B, \text { degree } q^{r+1}\right)
$$

where $y_{r}=a_{r} \tau^{r}\left(\omega^{r}\right)$ is a unit of $B$. It follows that $\mu_{B}$ maps the filtration subgroups $\omega^{r} B$ of $B$ into the filtration subgroups $C_{q}^{(r)}(F ; l)$ and that the induced maps

$$
l \stackrel{\sim}{\rightarrow} \omega^{r} B / \omega^{r+1} B \stackrel{\mu_{B}}{\rightarrow} C_{q}^{(r)}(F ; l) / C_{q}^{(r+1)}(F ; l) \stackrel{\sim}{\rightarrow} l
$$

are given by $x \mapsto y_{r} x^{q^{r}}$ for $x \in l$. (Here $l \stackrel{\sim}{\rightarrow} \omega^{r} B / \omega^{r+1} B$ is induced by $\omega^{r} b \mapsto \bar{b}$ with $\bar{b}$ the image of $b$ in $l$ under the canonical projection $B \rightarrow l$, and $C_{q}^{(r)}(F ; l) / C_{q}^{r+1}(F ; l) \stackrel{\sim}{\rightarrow} l$ is induced by $C_{q}^{(r)}(F ; l) \rightarrow l, \gamma(t) \mapsto\left(\right.$ coefficient of $t^{q^{r}}$ in $\gamma(t)$ ). Because $l$ is perfect and $\bar{y}_{r} \neq 0$, it follows that the induced maps $\bar{\mu}_{B}$ are all isomorphisms. Hence $\mu_{B}$ is an isomorphism because $B$ and $C_{q}(F ; l)$ are both complete in their filtration topologies. The map $\mu_{B}$ is an $A$-algebra homomorphism because $\Delta_{B}$ is an $A$-algebra homomorphism and $C_{q}(F ;-)$ is an $A$-algebra-valued functor. Finally the last statement of Theorem 6.6 follows because both $\tau$ and $\mu_{B}^{-1} \mathbf{f}_{\omega} \mu_{B}$ extend $\sigma$ and $\tau(b) \equiv b^{q} \equiv \mu_{B}^{-1} \mathbf{f}_{\omega} \mu_{B}(b) \bmod \omega B$.

6.7. The maps $s_{q, n}$ and $w_{q, n}^{F}$. The last thing to do is to reformulate the definitions of $C_{q}(F ; B)$ and $E_{B}$ in such a way that they more closely resemble the corresponding objects in the unramified case, i.e. in the case of the ordinary Witt vectors. This is easily done, essentially because $C_{q}(F ;-)$ is representable.

Indeed, let, as a set-valued functor, $W_{q, \infty}^{F}: \mathbf{A l g}_{A} \rightarrow$ Set be defined by

$$
\begin{gathered}
W_{q, \infty}^{F}(B)=\left\{\left(b_{0}, b_{1}, b_{2}, \ldots\right) \mid b_{i} \in B\right\}, \\
W_{q, \infty}^{F}(\phi)\left(b_{0}, b_{1}, \ldots\right)=\left(\phi\left(b_{0}\right), \phi\left(b_{1}\right), \ldots\right) .
\end{gathered}
$$


We now identify the set-valued functors $W_{q, \infty}^{F}(-)$ and $C_{q}(F ;-)$ by the functorial isomorphism

$$
e_{B}\left(b_{0}, b_{1}, \ldots\right)=\sum_{i=0}^{\infty}{ }^{F} b_{i} t^{q^{i}}
$$

and define $W_{q, \infty}^{F}(-)$ as an $A$-algebra-valued functor by transporting the $A$-algebra structure on $C_{q}(F ; B)$ via $e_{B}$ for all $B \in \mathbf{A l g}_{B}$. We use $f$ and $\mathrm{V}$ to denote the endomorphisms of $W_{q, \infty}^{F}(-)$ obtained by transporting $f_{\omega}$ and $V_{q}$ via $e_{B}$. Then one has immediately that

$$
\mathbf{V}\left(b_{0}, b_{1}, \ldots\right)=\left(0, b_{0}, b_{1}, \ldots\right)
$$

and in fact

$$
f\left(b_{0}, b_{1}, \ldots\right)=\left(\hat{b}_{0}, \hat{b}_{1}, \ldots\right) \Rightarrow \hat{b}_{i} \equiv b_{i}^{q} \bmod \omega B .
$$

(We have not proved the analog of this for $\mathbf{f}_{\omega}$; this is not difficult to do by using part (iv) of the Functional Equation Lemma and the additivity of $\mathbf{f}_{\omega}$.)

Next we discuss the analog of the Witt polynomials $X_{0}^{p^{n}}+p X_{1}^{p^{n-1}}$ $+\cdots+p^{n} X_{n}$. We define for the universal curve $\gamma_{T}(t) \in C_{q}(F ; A[T])$,

$$
s_{q, n}\left(\gamma_{T}(t)\right)=a_{n}^{-1}\left(\text { coefficient of } t^{q^{n}} \text { in } f\left(\gamma_{T}(t)\right)\right)
$$

and, as usual, this is extended functorially for arbitrary curves $\gamma(t)$ over arbitrary $A$-algebras by

$$
s_{q, n} \gamma(t)=\phi\left(s_{q, n}\left(\gamma_{T}(t)\right)\right)
$$

where $\phi: A[T] \rightarrow B$ is the unique $A$-algebra homomorphism such that $\phi_{*} \gamma_{T}(t)=$ $\gamma(t)$. If $B$ is $A$-torsion free one has, of course, the result that $s_{q, n} \gamma(t)=a_{n}^{-1}$ (coefficient of $t^{q^{n}}$ in $f(\gamma(t))$ ). Using this one checks that

$$
\begin{gathered}
s_{q, n}\left(\gamma(t)+{ }_{F} \delta(t)\right)=s_{q, n}(\gamma(t))+s_{q, n}(\delta(t)), \\
s_{q, n}(\gamma(t) * \delta(t))=s_{q, n}(\gamma(t)) s_{q, n}(\delta(t)), \\
s_{q, n}\left(\{a\}_{F} \gamma(t)\right)=\sigma^{n}(a) s_{q, n}(\gamma(t)), \\
s_{q, n}\left(f_{\omega} \gamma(t)\right)=s_{q, n+1}(\gamma(t)), \\
s_{q, n}\left(V_{q} \gamma(t)\right)=\sigma^{n-1}(\omega) s_{q, n-1}(\gamma(t)) \text { if } n>1, \\
s_{q, 0}\left(\mathbf{V}_{q} \gamma(t)\right)=0, \\
s_{q, n}(t)=1 \text { for all } n .
\end{gathered}
$$

Now suppose that we are in the situation of 6.1 above. Then, by the definition of $\Delta_{B}$, we have

$$
s_{q, n}\left(\Delta_{B}(b)\right)=\tau^{n}(b) .
$$

Now define $w_{q, n}^{F}(B): W_{q, \infty}^{F}(B) \rightarrow B$ by $w_{q, n}^{F}=s_{q, n} \circ e_{B}$. It is not difficult to calculate $w_{q, n}^{F}$. Indeed

$$
f\left(\gamma_{T}(t)\right)=f\left(\sum_{i=0}^{\infty} F T_{i} t^{q^{i}}\right)=\sum_{j=0}^{\infty} \sum_{i=0}^{\infty} a_{j} T_{i}^{j} t^{q^{i+1}}=\sum_{r=0}^{\infty}\left(\sum_{i=0}^{r} a_{i} T_{r-i}^{q^{\prime}}\right) t^{q^{\prime}}
$$


and it follows that $w_{q, n}^{F}$ is the functorial map $W_{q, \infty}^{F}(B) \rightarrow B$ defined by the polynomials

$$
\begin{aligned}
w_{q, n}^{F}\left(Z_{0}, \ldots, Z_{n}\right)= & a_{n}^{-1}\left(\sum_{i=0}^{n} a_{i} Z_{n-i}^{q^{i}}\right) \\
= & Z_{0}^{q^{n}}+\sigma^{n-1}(\omega) Z_{1}^{q^{n-1}}+\sigma^{n-1}(\omega) \sigma^{n-2}(\omega) Z_{2}^{q^{n-2}}+\cdots \\
& +\sigma^{n-1}(\omega) \cdots \sigma(\omega) \omega Z_{n} .
\end{aligned}
$$

6.17. TheOREM. Let $(A, \sigma)$ be a pair consisting of a discrete valuation ring $A$ of residue characteristic $p>0$ and a Frobenius-like automorphism $\sigma: K \rightarrow K$ such that (2.2) holds for some power $q$ of $p$. Let $\omega$ be any uniformizing element of $A$, and let $w_{q, n}^{F}(Z), n=0,1, \ldots$ be the polynomials defined by (6.16). Then there exists $a$ unique A-algebra-valued functor $W_{q, \infty}^{F}: \mathbf{A l g}_{A} \rightarrow \mathbf{A l g}_{A}$ such that

(i) as a set-valued functor $W_{q, \infty}^{F}(B)=\left\{\left(b_{0}, b_{1}, b_{2}, \ldots\right) \mid b_{i} \in B\right\}$ and $W_{q, \infty}^{F}(\phi)\left(b_{0}, b_{1}, \ldots\right)=\left(\phi\left(b_{0}\right), \phi\left(b_{1}\right), \ldots\right)$ for all $\phi: B \rightarrow B^{\prime}$ in $\mathbf{A l g}{ }_{A}$,

(ii) the polynomials $w_{q, n}^{F}(Z)$ induce functorial $\sigma^{n}$-semilinear A-algebra homomorphisms $w_{q, \infty}^{F}: W_{q, \infty}^{F}(B) \rightarrow B,\left(b_{0}, b_{1}, \ldots\right) \mapsto w_{q, n}^{F}\left(b_{0}, \ldots, b_{n}\right)$.

Moreover, the functor $W_{q, \infty}^{F}(-)$ has a $\sigma^{-1}$-semilinear $A$-module functor endomorphism $\mathbf{V}$ and a functorial $\sigma$-semilinear A-algebra endomorphism $\mathbf{f}$ which satisfy and are characterized by

(iii) $w_{q, n}^{F} \circ \mathrm{V}=\sigma^{n-1}(\omega) w_{q, n-1}^{F}$ if $n=1,2, \ldots ; w_{q, 0}^{F} \circ \mathrm{V}=0$,

(iv) $w_{q, n}^{F} \circ f=w_{q, n+1}^{F}$.

These endomorphisms f and $\mathrm{V}$ have (among others) the properties

(v) $\mathbf{f V}=\omega$,

(vi) $\mathbf{f} b \equiv b^{q} \bmod \omega W_{q, \infty}^{F}(B)$ for all $\mathbf{b} \in W_{q, \infty}^{F}(B), B \in \mathbf{A l g}_{A}$,

(vii) $\mathbf{V}(\mathbf{b}(\mathbf{f c}))=(\mathbf{V b}) \mathbf{c}$ for all $\mathbf{b}, \mathbf{c} \in W_{q, \infty}^{F}(B), B \in \mathbf{A l g}_{A}$.

Further there exists a unique functorial $A$-algebra homomorphism

$$
E: W_{q, \infty}^{F}(-) \rightarrow W_{q, \infty}^{F}\left(W_{q, \infty}^{F}(-)\right)
$$

which satisfies and is characterized by

(viii) $w_{q, n}^{F} \circ E=\mathbf{f}^{n}$ for all $n=0,1,2, \ldots$ (Here $w_{q, n}^{F}: W_{q, \infty}^{F}\left(W_{q, \infty}^{F}(B)\right) \rightarrow$ $W_{q, \infty}^{F}(B)$ is short for $w_{q, n, w_{q, \infty}^{F}(B)}^{F}$, i.e. it is the map which assigns to a sequence $\left(\mathbf{b}_{0}, \mathbf{b}_{1}, \ldots\right)$ of elements of $W_{q, \infty}^{F}(B)$ the element $w_{q, n}^{F}\left(b_{0}, b_{1}, \ldots\right) \in W_{q, \infty}^{F}(B)$.) The functor homomorphism $E$ further satisfies

(ix) $W_{q, \infty}^{F}\left(w_{q, n}^{F}\right) \circ E=\mathbf{f}^{n}$, where $W_{q, \infty}^{F}\left(w_{q, n}^{F}\right): W_{q, \infty}^{F}\left(W_{q, \infty}^{F}(B)\right) \rightarrow W_{q, \infty}^{F}(B)$ assigns to a sequence $\left(\mathrm{b}_{0}, \mathrm{~b}_{1}, \ldots\right)$ of elements of $W_{q, \infty}^{F}(B)$ the sequence $\left(w_{q, n}^{F}\left(b_{0}\right), w_{q, n}^{F}\left(b_{1}\right), \ldots\right)$ $\in W_{q, \infty}^{F}(B)$.

Finally if $A$ is complete with perfect residue field $k$ and $l / k$ is a finite separable extension, then $W_{q, \infty}^{F}(l)$ is the ring of integers $B$ of the unique unramified extension $L / K$ covering the residue field extension $l / k$ and under this $A$-algebra isomorphism $f$ corresponds to the unique extension of $\sigma$ to $\tau: B \rightarrow B$ which satisfies $\tau(b) \equiv b^{q}$ $\bmod \omega B$. In particular $W_{q, \infty}^{F}(k) \simeq A$ with $f$ corresponding to $\sigma$.

Proof. Existence of $W_{q, \infty}^{F}(-), \mathbf{V}, \mathbf{f}, E$ such that (i), (ii), (iii), (iv), (viii) hold follows from the constructions above. Uniqueness follows because (i), (ii), (iii), (iv), 
(viii) determine the $A$-algebra structure on $B^{\mathrm{N} \cup\{0\}}, \mathbf{V}, \mathbf{f}, E$ uniquely for $A$-torsion free $A$-algebras $B$, and then these structure elements are uniquely determined by (i)-(iv), (viii) for all $A$-algebras, by the functoriality requirement (because for every $A$-algebra $B$ there exists an $A$-torsion free $A$-algebra $B^{\prime}$ together with a surjective $A$-algebra homomorphism $B^{\prime} \rightarrow B$ ). Of the remaining identities some have already been proved in the $C_{q}(F ;-)$-setting ((v) and (vi)). They can all be proved by checking that they give the right answers whenever composed with the $w_{q, n}^{F}$. This proves that they hold over $A$-torsion free algebras $B$, and then they hold in general by functoriality. So to prove (vii) we calculate

$$
\begin{aligned}
w_{q, 0}^{F}(\mathbf{V}(\mathbf{b}(\mathbf{f c}))) & =0 \\
w_{q, n}^{F}(\mathbf{V}(\mathbf{b}(\mathbf{f c}))) & =\sigma^{n-1}(\omega) w_{q, n-1}^{F}(\mathbf{b}(\mathbf{f c}))=\sigma^{n-1}(\omega) w_{q, n-1}^{F}(\mathbf{b}) w_{q, n-1}^{F}(\mathbf{f c}) \\
& =\sigma^{n-1}(\omega) w_{q, n-1}^{F}(\mathbf{b}) w_{q, n}^{F}(\mathbf{c})
\end{aligned}
$$

and, on the other hand,

$$
\begin{aligned}
& w_{q, 0}^{F}((\mathbf{V b}) \mathbf{c})=w_{q, 0}^{F}(\mathbf{V b}) w_{q, 0}^{F}(\mathbf{c})=0, \quad w_{q, 0}^{F}(\mathbf{c})=0 \\
& w_{q, n}^{F}((\mathbf{V b}) \mathbf{c})=w_{q, n}^{F}(\mathbf{V b}) w_{q, n}^{F}(\mathbf{c})=\sigma^{n-1}(\omega) w_{q, n-1}^{F}(\mathbf{b}) w_{q, n}^{F}(\mathbf{c})
\end{aligned}
$$

This proves (vii). To prove (ix) we proceed similarly.

$$
w_{q, m}^{F} \circ W_{q, \infty}^{F}\left(w_{q, n}^{F}\right) \circ E=w_{q, n}^{F} \circ w_{q, m}^{F} \circ E=w_{q, n}^{F} \circ \mathbf{f}^{m}=w_{q, n+m}^{F}=w_{q, m}^{F} \circ \mathbf{f}^{n} .
$$

(Here the first equality follows from the functoriality of the morphisms $w_{q, m}^{F}$ which says that for all $\phi: B^{\prime} \rightarrow B \in \operatorname{Alg}_{A}$ we have $w_{q, m}^{F} \circ W_{q, \infty}^{F}(\phi)=\phi \circ w_{q, m}^{F}$; now substitute $w_{q, n}^{F}$ for $\left.\phi.\right)$

6.18. REMARK. Vf $=\mathrm{fV}$ does not, of course, hold in general (also not in the case of the usual Witt vectors). It is, however, true in $W_{q, \infty}^{F}(B)$ if $\omega B=0$, as easily follows from (6.11), which implies that $f\left(b_{0}, b_{1}, \ldots\right)=\left(b_{0}^{q}, b_{1}^{q}, \ldots\right)$ if $\omega B=0$.

\section{REFERENCES}

1. E. Artin and H. Hasse, Die beide Ergänzungssätze zum Reciprozitätsgesetz der $l^{n}$-ten Potenzreste im Körper der $l^{n}$-ten Einheitswürzeln, Abh. Math. Sem. Hamburg 6 (1928), 146-162.

2. P. Cartier, Groupes formels associés aux anneaux de Witt généralisés, C. R. Acad. Sci. Paris Sér. A-B 265 (1967), A49-A52.

3. Sér. A-B 265 (1967), A129-A132.

4. _ Relèvement des groupe formels commutatifs, Sem. Bourbaki (1968/1969), exposé 359, Lecture Notes in Math., no. 179, Springer-Verlag, Berlin and New York, 1971.

5. __ Séminaire sur les groupes formels, Inst. Hautes Études Sci., 1972 (unpublished notes).

6. J. Dieudonné, On the Artin-Hasse exponential series, Proc. Amer. Math. Soc. 8 (1957), 210-214.

7. E. J. Ditters, Formale Gruppen, die Vermutungen von Atkin-Swinnerton Dyer und verzweigte Witt-vektoren, Lecture Notes, Göttingen, 1975.

8. V. G. Drinfel'd, Coverings of p-adic symmetric domains, Funkcional. Anal. i Priložen. 10 (1976), 29-40. (Russian)

9. M. Hazewinkel, Une théorie de Cartier-Dieudonné pour les A-modules formels, C. R. Acad. Sci. Paris Sér. A-B 284 (1977), 655-657.

10. , “Tapis de Cartier” pour les A-modules formels, C. R. Acad. Sci. Paris Sér. A-B 284 (1977), 739-740. 
11. __ Formal groups and applications, Academic Press, New York, 1978.

12. J. Lubin and J. Tate, Formal complex multiplication in local fields, Ann. of Math. (2) 81 (1965), 380-387.

13. G. Whaples, Generalized local class field theory. III, Second form of existence theorem, Structure of analytic groups, Duke Math. J. 21 (1954), 575-581.

14. E. Witt, Zyklische Körper und Algebren der Characteristik p vom Grad p ${ }^{m}$, J. Reine Angew. Math. 176 (1937), 126-140.

Department of Mathematics, Erasmus University, 50, Burg. Oudlahn, Rotterdam, The NETHERLANDS 